\title{
DETERMINACIÓN DE LA CALIDAD DE LA PANELA GRANULADA ELABORADA EN EL RECINTO SAN CARLOS-JIPIJAPA.
}

AUTORES: Juan Miguel Garcia Cabrera ${ }^{1}$

Karla Victoria Chancay Ventura ${ }^{2}$

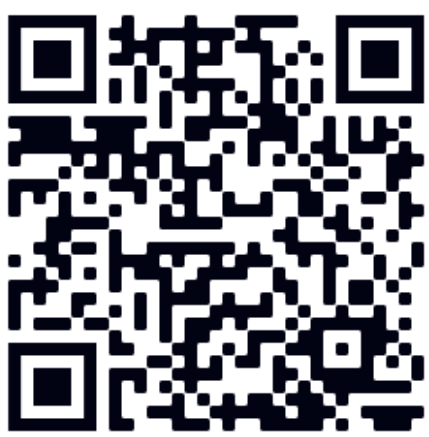

DIRECCIÓN PARA CORRESPONDENCIA:juanmiguel213@hotmail.com

Fecha de recepción: 03/05/2019

Fecha de aceptación: 08/07/2019

\section{RESUMEN}

Basado en las problemáticas agrícolas del recinto San Carlos en Jipijapa, se propuso generar una alternativa económica del procesamiento de la caña de azúcar; para obtener un derivado más de este producto como es el caso de la panela granulada, para el cual no solo fue necesario el requerimiento de un análisis bromatológico del producto, ya que de igual forma fue necesario realizar un estudio de prefactibilidad para determinar la viabilidad de producir en grandes volúmenes y lanzar al mercado este producto obtenido de la zona de estudio antes indicada. Fue posible determinar tanto los parámetros de calidad como de factibilidad utilizando la materia procesada y elaborada, la cual posteriormente fue enviada a un laboratorio bromatológico para identificar su calidad y composición; y para su competitividad con productos de iguales características existentes ya en el mercado, fue necesario aplicar técnicas de recolección de información a las personas que manejan este producto en el mercado y a quienes realizan el proceso de generación de panela granulada; así mismo es importante recalcar que no todos los productores elaboran panela granulada; por costos de producción, materiales, equipos y materia prima idónea para este proceso. De los resultados obtenidos tanto del análisis bromatológico como del estudio de prefactibilidad se determinó su viabilidad a la elaboración de este producto por los agricultores que se dedican a esta labor, mencionando que sería más rentable si se contara con apoyo de entidades gubernamentales y financieras; permitiendo un desarrollo económico y social a sus habitantes.

PALABRAS CLAVE: Panela granulada; Caña de azúcar; San Carlos

Determination of the quality of granulated panela produced at the San Carlos enclosureJipijapa.

\footnotetext{
${ }^{1}$ Docente de la carrera Agropecuaria. Universidad Estatal del Sur de Manabí

2 Economista Karla Victoria Chancay Ventura.
} 
Juan Miguel Garcia Cabrera, Karla Victoria Chancay Ventura, ...

\section{ABSTRACT}

Based on the agricultural problems of the San Carlos site in Jipijapa, it was proposed to generate an economic alternative to the processing of sugarcane; In order to obtain one more derivative of this product, such as granulated panela, for which the requirement of a bromatological analysis of the product was not only necessary, since a prefeasibility study was also necessary to determine the viability of Produce in large volumes and launch this product obtained from the study area indicated above. It was possible to determine both the quality and feasibility parameters using the processed and processed matter, which was subsequently sent to a bromatological laboratory to identify its quality and composition; and for its competitiveness with products of the same characteristics already existing in the market, it was necessary to apply information gathering techniques to the people who handle this product in the market and to those who carry out the process of generating granulated panela; It is also important to emphasize that not all producers produce granulated panela; for production costs, materials, equipment and raw material suitable for this process. From the results obtained from both the bromatological analysis and the prefeasibility study, its viability to the elaboration of this product was determined by the farmers who dedicate themselves to this work, mentioning that it would be more profitable if they had the support of governmental and financial entities; allowing economic and social development to its inhabitants.

KEYWORDS: Granulated Panela; Sugar cane; Saint Charles.

\section{INTRODUCCIÓN}

La caña de azúcar (Saccharum) es actualmente el cultivo agroalimentario más productivo del mundo. Debido a la naturaleza química y bioquímica de la materia prima que produce presenta gran potencial para la diversificación productiva.

Los desafíos que enfrenta actualmente la agroindustria panelera nacional requieren de reformas estructurales y coyunturales que permitan generar políticas públicas encaminadas a impulsar iniciativas de diversificación de los productos derivados de la caña de azúcar, actuales en mercados diversos, a través de estrategias eficientes, competitivas y amigables con el ambiente (Gómez, 2017). Entre la importancia podemos destacar, que el agro ecuatoriano es posible rescatarlo con una política de estado en beneficio de los agricultores; como créditos oportunos, acceso a tecnología, políticas que ayuden en el almacenamiento de las cosechas, capacitación sobre comercialización de sus productos, generación de asociaciones agro productivas, entre otras. Que permitirán una competitividad de los campesinos y agricultores frente a las grandes industrias, que muchas veces son aprovechadas de los pocos conocimientos que tienen estas personas ante estos sectores.

Según estudios se ha podido identificar que en el mundo se estiman cerca de 25 millones de sembradas de caña de azúcar, principalmente para extracción de azúcar. Las diferentes industrias del planeta promueven más de 300 millones de empleos directos por año. Y en Ecuador se cosechan anualmente aproximadamente 81.000 hectáreas de caña azúcar para producción de azúcar blanca y etanol. Otras 50.000 hectáreas ha se destinan para producción de panela y alcohol artesanal (Zambrano, 2016). Lo antes indicado permitió determinar la calidad de la panela granulada elaborada en el recinto San Carlos de Jipijapa, lo cual conllevaría a un desarrollo no solamente 
económico, sino también turístico y con un crecimiento social entre sus habitantes que solo se dedican a las prácticas agrícolas y pecuarias para su subsistencia.

\section{DESARROLLO}

\section{Metodología}

Para el desarrollo de esta investigación fue necesario el empleo de una serie de métodos los cuales permitieron realizar comparaciones con bibliografías relacionadas a este proceso, no solo literariamente sino también de valores de composición y calidad de la panela granulada; y así mismo se realizaron entrevistas y encuestas tanto a productores, consumidores y comercializantes de panela granulada en la zona y en la ciudad de Jipijapa; de la misma manera se ejecutó un análisis bromatológico el cual permitió identificar el contenido de vitaminas, grasas, minerales entre otros parámetros que demuestran su composición y calidad para el consumo de este producto; de esta forma también fue posible realizar un análisis de prefactibilidad el cual arrojo la viabilidad en la generación y comercialización de la panela granulada como una nueva alternativa económica para la subsistencia de sus productores y el desarrollo de la zona.

\section{Resultados}

Para determinar la muestra de la población de 71.083 habitantes en la ciudad de Jipijapa respecto a los datos obtenidos del Instituto Nacional de Estadísticas y Censos (INEC).

$$
\mathrm{n}=\frac{Z^{2}(P * Q)(N)}{\left(e^{2}\right)(N)+\left(Z^{2}\right)(P * Q)}
$$

$\mathbf{n}=$ Tamaño de la muestra

$\mathbf{P} * \mathbf{Q}=0,25$

$\mathbf{Z}^{\mathbf{2}}=$ Margen de confiabilidad. $(1,96)$

$\mathbf{e}=$ Error admisible $(0,05)$

$\mathbf{N}=$ Tamaño de la población (71.083)

$$
\begin{gathered}
\mathrm{n}=\frac{(1,96)^{2}(0,25)(71.083)}{\left(0,05^{2}\right)(71.083)+\left(1,92^{2}\right)(0,25)}=\frac{(3,84)(0,25)(71.083)}{(0,0025)(71.083)+(3,84)(0,25)} \\
n=\frac{68.239}{177,71+0,96}=\frac{68.239}{178,67}=382
\end{gathered}
$$

Donde, se obtuvo una población de 382 personas las cuales fueron encuestadas.

\section{Análisis Bromatológico:}

Tabla 1: Nutricional de la panela

\begin{tabular}{|c|c|c|c|}
\hline Análisis & $\begin{array}{c}\text { Análisis Bromatológico } \\
\text { Agrocalidad }\end{array}$ & $\begin{array}{c}\text { Límite } \\
\text { inferior }\end{array}$ & $\begin{array}{c}\text { Limite } \\
\text { Superior }\end{array}$ \\
\hline Humedad \% & 6,45 & 5,77 & 10,18 \\
\hline
\end{tabular}


Juan Miguel Garcia Cabrera, Karla Victoria Chancay Ventura, ...

\begin{tabular}{|c|c|c|c|}
\hline Proteína \% & 0,80 & 0,39 & 1,13 \\
\hline Grasa \% & 0,17 & 0,13 & 0,15 \\
\hline Cenizas \% & 0,65 & 0,61 & 1,36 \\
\hline Fibra\% & 0,11 & 0,24 & 0,24 \\
\hline \multicolumn{3}{|c|}{ Minerales mg/ 100g } \\
\hline pH \% & 5,69 & 5,77 & 6,17 \\
\hline Calcio & 0,04 & 0,57 & 4,72 \\
\hline Potasio & 0,18 & 0,59 & 3,66 \\
\hline Hierro & $<0,5$ & 0,022 & 0,08 \\
\hline
\end{tabular}

Fuente: Determinación de la composición físico-química de la panela

Elaborado por: Autor de la investigación

Estudio de prefactibilidad:

Tabla 2: Flujo de Caja

\begin{tabular}{|c|c|c|c|c|c|c|}
\hline INGRESOS & AÑO 0 & AÑ̃ 1 & AÑO 2 & AÑ̃ 3 & AÑO 4 & AÑO 5 \\
\hline Ventas netas & & $\$ 265.890,15$ & $\$ 279.344,19$ & $\$ 293.479,01$ & $\$ 308.329,05$ & $\$ 323.930,50$ \\
\hline TOTAL INGRESOS & & $\$ 265.890,15$ & $\$ 279.344,19$ & $\$ 293.479,01$ & $\$ 308.329,05$ & $\$ 323.930,50$ \\
\hline \multicolumn{7}{|c|}{ COSTOS DE PRODUCCIÓN } \\
\hline Materia prima & & $\$ 144.000,00$ & $\$ 146.880,00$ & $\$ 149.817,60$ & $\$ 152.813,95$ & $\$ 155.870,23$ \\
\hline Mano de obra directa & & $\$ \quad 29.040,00$ & $\$ 29.620,80$ & $\$ 30.213,22$ & $\$ \quad 30.817,48$ & $\$ 31.433,83$ \\
\hline Costos indirectos & & $\$ \quad 30.304,25$ & $\$ 30.910,34$ & $\$ 31.528,54$ & $\$ \quad 32.159,11$ & $\$ 32.802,29$ \\
\hline Depreciación & & $\$ \quad 3.139,25$ & $\$ \quad 3.139,25$ & $\$ 3.139,25$ & $\$ \quad 3.139,25$ & $\$ \quad 3.139,25$ \\
\hline TOTAL COSTO DE PRODUCCIÓN & & $\$ 206.483,50$ & $\$ 210.550,39$ & $\$ 214.698,61$ & $\$ 218.929,79$ & $\$ 223.245,61$ \\
\hline \multicolumn{7}{|c|}{ GASTOS OPERACIONALES } \\
\hline Gastos administrativos & & $\$ 14.083,10$ & $\$ 14.364,76$ & $\$ 14.652,06$ & $\$ \quad 14.945,10$ & $\$ 15.244,00$ \\
\hline Gasto de ventas & & $\$ 15.042,93$ & $\$ 15.343,79$ & $\$ 15.650,67$ & $\$ \quad 15.963,68$ & $\$ 16.282,95$ \\
\hline Depreciación & & $\$ 1.445,07$ & $\$ 1.445,07$ & $\$ 1.445,07$ & $\$ \quad 1.178,40$ & $\$ 1.178,40$ \\
\hline Amortización & & 451,60 & $\$ \quad 451,60$ & $\$ \quad 451,60$ & 451,60 & $\$ \quad 451,60$ \\
\hline TOTAL GASTOS OPERACIONALES & & $\$ 31.022,70$ & $\$ 31.605,22$ & $\$ 32.199,39$ & $\$ 32.538,78$ & $\$ 33.156,96$ \\
\hline $\begin{array}{l}\text { TOTAL COSTOS Y GASTOS } \\
\text { OPERACIONALES }\end{array}$ & & $\$ 237.506,20$ & $\$ 242.155,61$ & $\$ 246.898,00$ & $\$ 251.468,57$ & $\$ 256.402,56$ \\
\hline UTILIDAD OPERACIONAL & & $\$ 28.383,95$ & $\$ 37.188,59$ & $\$ 46.581,01$ & $\$ 56.860,47$ & $\$ 67.527,94$ \\
\hline GASTOS FINANCIEROS & & $\$$ & $\$$ & $\$$ & $\$$ & $\$$ \\
\hline Intereses pagados & & $\$ 3.218,65$ & $\$ 2.658,69$ & $\$ 2.041,33$ & $\$ \quad 1.360,69$ & $\$ \quad 610,28$ \\
\hline TOTAL GASTOS FINANCIEROS & & $\$ 3.218,65$ & $\$ 2.658,69$ & $\$ \quad 2.041,33$ & $\$ \quad 1.360,69$ & $\$ \quad 610,28$ \\
\hline Utilidad antes de imp. Y part. & & $\$ 25.165,30$ & $\$ 34.529,90$ & $\$ 44.539,68$ & $\$ 55.499,79$ & $\$ 66.917,66$ \\
\hline $15 \%$ participación trabajadores & & $\$ 3.774,79$ & $\$ 5.179,48$ & $\$ 6.680,95$ & $\$ \quad 8.324,97$ & $\$ 10.037,65$ \\
\hline $25 \%$ impuesto a la renta & & $\$ 5.347,63$ & $\$ 7.337,60$ & $\$ 9.464,68$ & $\$ 11.793,70$ & $\$ 14.220,00$ \\
\hline
\end{tabular}

12 UNESUM-Ciencias. Publicación cuatrimestral. Vol. 3, Año 2019, No. 2 (Mayo - Agosto) 
UNESUM-Ciencias: Revista Científica Multidisciplinaria

ISSN 2602-8166

DETERMINACIÓN DE LA CALIDAD DE LA PANELA GRANULADA

\begin{tabular}{|c|c|c|c|c|c|c|c|}
\hline \multicolumn{2}{|l|}{ UTILIDAD NETA } & & $\$ 16.042,88$ & $\$ 22.012,81$ & $\$ 28.394,05$ & $\$ 35.381,11$ & $\$ 42.660,01$ \\
\hline \multicolumn{2}{|l|}{ Depreciación } & & $\$ 4.584,32$ & $\$ 4.584,32$ & $\$ 4.584,32$ & $\$ \quad 4.317,65$ & $\$ 4.317,65$ \\
\hline \multicolumn{2}{|l|}{ Amortización } & & $\$ \quad 451,60$ & 451,60 & 451,60 & 451,60 & $\$ \quad 451,60$ \\
\hline \multicolumn{2}{|l|}{ Inversiones en activo } & $\$(53.085,75)$ & & & & & \\
\hline \multicolumn{2}{|l|}{ Capital de trabajo } & $\$(19.874,97)$ & $\$(4.649,41)$ & $\$(4.742,39$ & $\$(4.570,57)$ & $\$(4.933,99)$ & $\$ 256.402,56$ \\
\hline \multirow{2}{*}{\multicolumn{2}{|c|}{ Valor de desecho }} & & & & & & $\$ 18.989,50$ \\
\hline & & $\$(72.960,72)$ & $\$ \quad 16.429,39$ & $\$ \quad 22.306,33$ & $\$ 28.859,39$ & $\$ \quad 35.216,38$ & $\$ 322.821,32$ \\
\hline & \multicolumn{4}{|l|}{ VAN } & $\$ 185.590,52$ & & \\
\hline & \multicolumn{4}{|l|}{ TIR } & $53 \%$ & & \\
\hline & \multicolumn{4}{|c|}{ COSTO BENEFICIO } & $\$ 3,54$ & & \\
\hline
\end{tabular}


Juan Miguel Garcia Cabrera, Karla Victoria Chancay Ventura, ...

\section{Discusión}

De acuerdo a los resultados la producción de caña genera ingresos a sus productores, de acuerdo a la investigación realizada por (Herrera Suárez, 2015) señala:

El cultivo de la caña de azúcar ha tenido una gran trascendencia en la economía del sector agropecuario en Ecuador, pues no solo forma parte de uno de los eslabones más importante de las cadenas agroproductivos, si no, que sirve de sustento a un grupo numeroso de familias, siendo una importante fuente de generación de empleos, con una importante contribución al PIB agrícola nacional del 12\%. (El Telegrafo, 2016) Hace referencia a que se investigan 100 parcelas de cultivo de la zona de San Carlos, área rural de Jipijapa. En la zona se trabaja con 22 productores. Este punto es el de mayor producción, entre 75 y 90 toneladas por hectárea. De la misma manera se hace énfasis al estudio realizado por el ( Ministerio de Defenza Nacional, 2012), acuerdo a la dinámica local observada, en este cantón es posible identificar que todos los productos agrícolas tienen un objetivo comercial, sin embargo, el productor separa una porción de la cosecha para el consumo familiar. Según (Daza, 2014), el gobierno ecuatoriano ha priorizado la trasformación de la Matriz Productiva como una de las principales vías para la sustitución selectiva de importaciones. ...Este proceso pretende el mejoramiento de los rendimientos agrícolas de los cultivos a partir de una intensificación productiva, ya sea mediante la introducción de paquetes tecnológicos o el incremento de las áreas productivas.

Al transformar la materia prima (caña de azúcar), nos sirve exclusivamente para producir aguardiente, alfeñique y panela permitiéndole cumplir con sus compromisos adquiridos hacia sus clientes. Según (El Diario, 2018), uno de los clientes fijos por más de 30 años es Don Panchito ya tiene 86 años y cuenta, feliz, que desde hace 70 se dedica a vender derivados de la caña de azúcar, ahora solo en Jipijapa. Los productos los obtiene en el sector San Carlos, donde se dedican al cultivo de caña y donde él nació.

La producción de la panela granulada con lleva un sin números de procesos dentro del cual (Panduro Alegria, 2007), manifiesta que el proceso es igual hasta llegar al proceso de cristalizado, se deja enfriar durante un lapso de media hora, para evitar que el azúcar se compacte. El azúcar ecológico es pasado por un tamiz de acero inoxidable que posee orificios de 2 milímetros, los cristales mayores a este diámetro ingresan nuevamente al proceso (a la paila evaporadora). Mediante estudio realizado por (Jaramillo Martinez \& Vargas Moreira, 2012), en la ciudad de Milagro, manifiestan en su impacto, que la implementación de la micro empresa tendrá un alto nivel de aceptabilidad contribuyendo de forma directa al consumo natural.

... el negocio muestra rentabilidad independiente de la competencia, sin embargo, el logro de los resultados del proyecto demuestra un buen manejo administrativo de la comercializadora además de la aplicación de estrategias que potencien su posicionamiento en el mercado, creando ventajas competitivas que impulsan su desarrollo permanente.

\section{CONCLUSIONES}

Se pudo evidenciar según las técnicas aplicadas para la recopilación de datos dirigida a los microempresarios, que los derivados de la caña de azúcar que se producen en el recinto San Carlos generan una gran fuente de ingresos para ellos; debido a que su subsistencia principal es la actividad agroproductiva elaborada y procesada a partir de esta planta. 
Mediante el estudio de pre factibilidad fue posible determinar que la viabilidad de este proyecto es rentable, con cálculos de un VAN \$185.590,52; un TIR del 53\% y el costo beneficio de \$3,54 de la cual se basa en la producción y comercialización a gran escala de la panela granulada, la cual podría ser un reemplazo saludable y económico con relación al azúcar blanco que es utilizado comúnmente en los hogares como endulzante.

La panela granulada es considerada rentable según el estudio de pre factibilidad, al igual que la elaboración del aguardiente que es otro de los derivados de la caña de azúcar y de los productos que se elaboran en el recinto San Carlos con un rubro económico de igual acogida.

\section{REFERÉNCIAS BIBLIOGRÁFICAS}

Ministerio de Defenza Nacional. (2012). www.app.sni.gob.ec. Obtenido de http://app.sni.gob.ec/snilink/sni/PDOT/ZONA4/NIVEL_DEL_PDOT_CANTONAL/MANABI/JIPIJAPA/IEE/MEM ORIAS_TECNICAS/mt_jipijapa_socioeconomico.pdf

Andres Montoyo, M. M. (2012). Proceso de produccion. Obtenido de https://rua.ua.es/dspace/bitstream/10045/19047/1/Tema_4_-_Proceso_de_produccion.pdf

Biografias y Vidas. (2004). www.biografiasyvidas.com. Obtenido de https://www.biografiasyvidas.com/biografia/l/lewis_william.htm

Buele, J. C. (2017). Comercialización de panela y su aporte al desarrollo socioeconomico. Obtenido de http://repositorio.unesum.edu.ec/bitstream/53000/1162/1/UNESUMECUADOR-COMERCIO\%2OEXT.2018-02.pdf

Castillo Martín, P. (2011). Política económica. Revista Internacional del Mundo Económico y del Derecho Volumen III, 2. Obtenido de http://www.revistainternacionaldelmundoeconomicoydelderecho.net/wpcontent/uploads/RIMED-Pol\%C3\%ADtica-econ\%C3\%B3mica.pdf

Cidecolombia. (2018). www.cidecolombia.com. Obtenido de https://cidecolombia.com/que-es-lapanela-y-beneficios-de-la-panelal

Daza, E. (2014). https://lalineadefuego.info. Obtenido de https://lalineadefuego.info/2014/12/16/transformacion-productiva-estado-y-agronegociocoyuntura-agraria-2013-2014-por-estaban-dazal

El Diario. (22 de septiembre de 2018). www.eldiario.ec. Obtenido de http://www.eldiario.ec/noticias-manabi-ecuador/483319-panchito-endulza-la-vida-en-elsurl

El Telegrafo. (12 de marzo de 2016). www.eltelegrafo.com.ec. Obtenido de https://www.eltelegrafo.com.ec/noticias/regional/1/prometeo-realiza-estudio-sobre-lacana-de-azucar-para-potenciar-su-produccion

FAO. (2015). www.fao.org. Obtenido de http://www.fao.org/3/Y0491s/y0491s02.htm

FAO. (2016). Agroindustria panelera en el ecuador. Obtenido de http://repositorio.utn.edu.ec/bitstream/123456789/350/2/03\%20AGI\%20201\%20CAPITU LO\%20II\%20\%20MARCO\%20TEORICO.pdf 
Juan Miguel Garcia Cabrera, Karla Victoria Chancay Ventura, ...

Garcia González, F. (octubre de 2012). Conceptos sobre innovación. Obtenido de https://www.acofi.edu.co/wpcontent/uploads/2013/08/DOC_PE_Conceptos_Innovacion.pdf

Gómez, M. (Noviembre de 2017). La diversificaciòn de la agroindustria azucarera como estrategia para Mexico. Agroproductividad, 10, 9. Obtenido de https://www.colpos.mx/wb_pdf/Agroproductividad/2017/AP-10-11-2017_ISSN-e.pdf

Gutierrez Garza, E. (2007). Trayectorias. Obtenido de https://www.redalyc.org/pdf/607/60715120006.pdf

Herrera Suárez, M. (2015). www.educacionsuperior.gob.ec. Obtenido de http://repositorio.educacionsuperior.gob.ec/bitstream/28000/4954/2/ANEXO\%202\%20C A\%C3\%91A\%20DE\%20AZUCAR.pdf

Jaramillo Martinez, P. R., \& Vargas Moreira, G. M. (2012). Estudio de Factivilidad paara la creacion de una comercializadora de panela granulada. Milagro: Universidad Estatal de Milagro.

Moreno Loza, G., \& Torres Salazar, S. (noviembre de 2011). Propuesta para la creación de una microempresa comunitaria asociativa de producción, industrialización comercialización de panela orgánica granulada. Obtenido de http://repositorio.puce.edu.ec/bitstream/handle/22000/4439/Tesis\%20Gabriela\%20More no\%20y\%20Sandra\%20Torres.pdf? sequence $=3 \&$ isAllowed $=y$

Panduro Alegria, J. C. (2007). www.urp.edu.pe. Obtenido de http://repositorio.urp.edu.pe/bitstream/handle/urp/177/panduro_jc.pdf?sequence $=1 \& i s A l$ lowed $=y$

Pantoja, J., \& Arévalo, E. (2006). La empresa moderna en el marco de la corriente institucionalista. Revista EIA. Obtenido de http://www.scielo.org.co/scielo.php?script=sci_arttext\&pid=S1794-12372006000100006

Porras Medina, A. A. (2018). Rediseño de un programa de identidad corporativa y campaña publicitaria de lanzamiento para la empresa agroindustrial panelera el valle. Obtenido de http://dspace.espoch.edu.ec/bitstream/123456789/9166/1/88T00261.pdf

Quezada, W. (2007). http://repositorio.utn.edu.ec. Obtenido de http://repositorio.utn.edu.ec/bitstream/123456789/934/1/Gu\%C3\%ADa\%20T\%C3\%A9cn ica\%20de\%20Agroindustria\%20Panelera.pdf

Reyes Alvarez, S. M., \& Silva Jara, J. G. (2009). Creación de una microempresa dedicada a la elaboracion y comercializacion de panela granulada en la ciudad de milagro. Obtenido de http://www.panelamonitor.org/media/docrepo/document/files/creacion-de-unamicroempresa-dedicada-a-la-elaboracion-y-comercializacion-de-panela-granulada.pdf

Romero, E. (2012). La caña de azúcar Características y ecofisiología. Obtenido de http://www.eeaoc.org.ar/upload/publicaciones/archivos/52/20120305122908000000.pdf

Stanton. (1 de 11 de 2014). Comercializacion. Obtenido de https://es.scribd.com/document/329639849/Comercializacion-Citas-Segun-Autores 
Zambrano, A. (2016). Investigaciones del CINCAE aumentan productividad en caña de azùcar. El Agro. Obtenido de http://www.revistaelagro.com/2016/02/01/investigaciones-del-cincaeaumentan-productividad-en-caña-de-azucarl 
«Autor1», «Autor2», «Autor3»...

18 UNESUM-Ciencias. Publicación cuatrimestral. Vol. 3, Año 2019, No. 2 (Mayo - Agosto) 